\title{
Avaliação antropométrica da população escolar do concelho de Boticas
}

José Alves, ${ }^{*}$ Julieta Gomes, ${ }^{* *}$ Marta Santos, ${ }^{* * *}$ Maria de Deus Barroso****

\section{RESUMO}

Objectivos: Determinar a prevalência de baixo peso, excesso de peso, obesidade, risco de obesidade abdominal e obesidade abdominal estabelecida na população escolar do concelho de Boticas.

Tipo de estudo: Estudo observacional, transversal.

Local: Estabelecimentos escolares do concelho de Boticas.

População: A totalidade dos alunos do concelho de Boticas frequentando entre $\circ 1^{\circ}$ e o $9^{\circ}$ ano de escolaridade durante o ano lectivo de 2012-2013.

Métodos: Determinação do peso, da estatura e do índice de massa corporal dos alunos e sua comparação com as curvas de crescimento preconizadas pela Direcção Geral da Saúde; determinação do perímetro da cintura e sua comparação com a tabela de Fernandez e col., como proposto também pela DGS.

Resultados: No ano lectivo de 2012-2013 frequentaram o Agrupamento de Escolas Gomes Monteiro, em Boticas, 372 alunos. Verificou-se excesso de peso em $19,7 \%$ dos alunos com predomínio do sexo masculino e obesidade em $12,1 \%$ dos alunos com predomínio do sexo feminino. Verificou-se risco de obesidade abdominal e obesidade abdominal estabelecida em $45,1 \%$ dos alunos com predomínio do sexo feminino.

Conclusões: A obesidade infantil representa um problema de saúde importante no concelho de Boticas, com uma prevalência semelhante à descrita na literatura científica. O estudo poderá ser o ponto de partida para o desenvolvimento de programas de intervenção na obesidade infantil.

Palavras-chave: Excesso de Peso; Obesidade Infantil; Índice de Massa Corporal; Perímetro da Cintura.

\section{INTRODUÇÃO}

A avaliação estatoponderal é uma actividade essencial das consultas de saúde infanto-juvenil, considerando-se que o crescimento é um importante indicador do bem-estar de uma criança ou adolescente. ${ }^{1}$

Dessas actividades de vigilância resulta uma apreciação que importa confrontar com o conhecimento exacto da realidade.

\footnotetext{
*Médico Assistente Graduado de Clínica Geral - Centro de Saúde de Boticas, ACES Alto Tâmega e Barroso

**Médica Interna de Medicina Geral e Familiar - Centro de Saúde de Boticas, ACES Alto Tâmega e Barroso

***Médica Interna de Medicina Geral e Familiar - Centro de Saúde de Boticas, ACES Alto Tâmega e Barroso

****Enfermeira Especialista - Centro de Saúde de Boticas, ACES Alto Tâmega e Barroso
}

Recentemente, o combate ao excesso de peso e a obesidade têm sido uma preocupação crescente de todos os profissionais que trabalham com as crianças e suas famílias. A obesidade é já considerada a pandemia do século XXI, ${ }^{2}$ sendo Portugal um dos países europeus onde este problema é mais evidente. ${ }^{3}$ De facto, estima-se que cerca de $30 \%$ das crianças portuguesas apresentem peso excessivo e, destas, 10 a $15 \%$ sejam obesas. ${ }^{4-10} \mathrm{~A}$ obesidade pediátrica tende a prolongar-se na idade adulta, sendo unanimemente aceite como factor de risco de múltiplas perturbações de ordem física, psíquica e social., ${ }^{211-12}$ Depende de factores tão diversos como a hereditariedade, os estilos de vida ou uma longa lista de possíveis doenças, exigindo muitas vezes uma abordagem multidisciplinar para a sua correcção. A importância deste problema, dada a sua magnitude e as suas consequências, contrasta com a dificuldade, 
já demonstrada, que os profissionais de saúde e os pais e educadores sentem no seu reconhecimento e detecção precoces. ${ }^{13-16}$

No entanto, estão hoje disponíveis intervenções com efeitos comprovados na prevenção e no tratamento da obesidade infantil e juvenil. ${ }^{17-23}$ Sabe-se também que tais intervenções podem reduzir ou até reverter o impacto deste problema. ${ }^{11,24-26}$ Esta possibilidade de actuação torna pertinente o incremento notável que os estudos nesta área têm verificado. A Plataforma Contra a Obesidade reconhece "a necessidade de intensificar trabalhos de investigação científica e aplicada” nesta área e preconiza, entre as suas estratégias de actuação, a promoção da avaliação das medidas antropométricas, nomeadamente peso, estatura, índice de massa corporal e perímetro da cintura. ${ }^{27-28}$ Estas medidas são de execução rápida, fácil e não dispendiosa e, sendo observadas metodologias e técnicas correctas e internacionalmente recomendadas, permitem uma avaliação rigorosa e comparável do estado nutricional. ${ }^{29-30}$

Por outro lado, o Programa Nacional de Saúde Escolar $^{31}$ estabelece que os projectos a desenvolver a nível local no âmbito deste Programa devem ter em conta as prioridades nacionais para as áreas de promoção de estilos de vida saudáveis e prevenção de comportamentos nocivos, mas devem também ser dirigidos para as necessidades de cada comunidade escolar. Assim, a elaboração desses projectos tem na sua base a identificação e caracterização de tais necessidades. Nesse sentido, a Equipa de Saúde Escolar do Centro de Saúde de Boticas colaborou no Plano Anual de Actividades 2012-2013 do Agrupamento de Escolas Gomes Monteiro no concelho de Boticas para, entre outras actividades, identificar crianças em situação de doença ou de risco relacionado com o seu estado nutricional.

Aproveitando a existência desses dados no centro de saúde, um grupo de profissionais decidiu que seria vantajoso fazer um tratamento dos dados que permitisse uma compreensão mais abrangente da situação. Com este estudo pretendeu-se fazer a avaliação antropométrica da população escolar do concelho de Boticas, tendo como objectivos específicos a determinação da prevalência de baixo peso, de excesso de peso, de obesidade, de pré-obesidade abdominal e de obesidade abdominal nos alunos do Agrupamento de Escolas Gomes
Monteiro que frequentaram entre o $1^{\circ}$ e o $9^{\circ}$ ano de escolaridade durante o ano lectivo de 2012-2013.

\section{MÉTODOS}

Procedeu-se a um estudo de tipo observacional, transversal, obedecendo às seguintes orientações:

\section{População em estudo}

A população alvo foi a totalidade da população escolar do Agrupamento de Escolas Gomes Monteiro. frequentando entre o $1^{\circ}$ e o $9^{\circ}$ ano de escolaridade, durante o ano lectivo de 2012-2013. Este Agrupamento é o único no concelho de Boticas e não havia crianças com residência no concelho frequentando estabelecimentos de ensino noutros concelhos neste nível de escolaridade. Foi critério de exclusão a ausência de registo, no Centro de Saúde, das variáveis em estudo.

\section{Variáveis em estudo}

As variáveis em estudo foram:

- Sexo-masculino ou feminino;

- Idade - número de anos concluídos à data das medições;

- Peso - em quilogramas;

- Estatura-em centímetros;

- Índice de massa corporal (IMC) - valor obtido pela divisão do peso, em quilogramas, pelo quadrado da estatura, em metros;

- Perímetro da cintura - em centímetros.

\section{Métodos de Medição}

Os registos foram efectuados no âmbito das actividades de Saúde Escolar do Centro de Saúde de Boticas, cumprindo as orientações do Guia da Avaliação do Estado Nutricional Infantil e Juvenil do Ministério da Saúde: 29

Peso - As crianças foram pesadas de manhã, com roupa leve (calças/calções ou saia e camisa ou blusa), sem sapatos, em balança electrónica, sendo considerado o valor em quilogramas, aproximados à décima.

Estatura - $\mathrm{O}$ valor da estatura foi verificado imediatamente a seguir ao peso. Foi utilizado um estadiómetro montado num ângulo de $90^{\circ}$ com o chão (apoiado contra uma parede). $\mathrm{O}$ valor da estatura foi registado em centímetros até à décima.

Perímetro da cintura - Usando uma fita métrica gra- 
duada ao milímetro, de material flexível mas não extensível. A medição foi obtida em pé, sobre a pele, no final de uma expiração normal, com a fita métrica na horizontal, ao nível das cristas ilíacas.

As medições foram realizadas em Maio de 2013 nas instalações escolares, por enfermeiras e alunas de Enfermagem em estágio no Centro de Saúde. Sempre que houve motivo para dúvidas procedeu-se à repetição das medições.

\section{Análise estatística}

Como medidas estatísticas foram utilizadas as frequências absolutas (n) e relativas (\%) das variáveis estudadas.

O peso, a estatura e o IMC foram avaliados por comparação com as curvas de crescimento preconizadas pela Direcção Geral da Saúde. ${ }^{1,29}$ O perímetro da cintura foi avaliado por comparação com a tabela de Fernandez e col., como proposto também pela Direcção Geral da Saúde. ${ }^{32}$

Foi considerada população escolar com baixo peso a que apresentou IMC no percentil inferior a 5 para a idade e sexo.

Foi considerada população escolar com excesso de peso a que apresentou IMC entre os percentis 85 e 95 para a idade e sexo.

Foi considerada população escolar com obesidade a que apresentou IMC no percentil superior a 95 para a idade e sexo.

Ao conjunto de excesso de peso e obesidade foi atribuída a designação de peso excessivo.

Foi considerada população escolar com aumento de risco de obesidade abdominal a que apresentou um perímetro da cintura no percentil superior ou igual a $75 \mathrm{e}$ inferior a 90 para a idade e sexo.

Foi considerada população escolar com obesidade abdominal a que apresentou um perímetro da cintura no percentil igual ou superior a 90 para a idade e sexo.

No tratamento dos dados recorreu-se à aplicação informática Excel®.

\section{RESULTADOS}

No ano lectivo de 2012-2013 frequentaram o Agrupamento de Escolas Gomes Monteiro, em Boticas, 372 alunos, 187 do sexo masculino e 185 do sexo feminino, distribuídos por idade e por sexo como se expõe no
Quadro I.

Não foi possível avaliar o peso, a estatura e o perímetro da cintura num aluno com 11 anos de idade por ser portador de deficiência que implicava a sua permanência numa cadeira de rodas. Também por deficiência física não foi possível avaliar o perímetro da cintura numa aluna com 7 anos de idade.

As variáveis peso e estatura apresentaram uma distribuição semelhante. Verificou-se um pico de maior frequência nos percentis centrais $(25<50,50<75 \mathrm{e}$ $75<90$ ), responsáveis por $64,4 \%$ do total em relação ao peso e por $67,1 \%$ em relação à estatura e um segundo pico no percentil > 95 (14,0\% em relação ao peso e 9,4\% em relação à estatura). Essa variação era semelhante em ambos os sexos.

A Figura 1 mostra a distribuição da população escolar segundo as classes do índice de massa corporal. Dos 371 alunos com avaliação do IMC identificaram-se 8 alunos $(2,2 \%)$ com baixo peso e 126 alunos $(34,0 \%)$ com peso excessivo, sendo que destes 64 eram do sexo masculino e 62 eram do sexo feminino. Revelaram obesidade 51 alunos, o que correspondeu a 13,7\% do total e a $40,5 \%$ dos alunos com peso excessivo.

$\begin{aligned} & \text { QUADRO I. População escolar: distribuição por idade e } \\
& \text { sexo }\end{aligned}$
\begin{tabular}{l|c|c|c|} 
Idade & Sexo & Sexo & \\
(anos) & Masculino & Feminino & TOTAL \\
\hline 6 & 19 & 8 & 27 \\
7 & 17 & 22 & 39 \\
8 & 19 & 21 & 40 \\
9 & 23 & 20 & 43 \\
10 & 20 & 19 & 39 \\
11 & 18 & 22 & 40 \\
12 & 11 & 20 & 31 \\
13 & 20 & 19 & 39 \\
14 & 19 & 16 & 35 \\
15 & 13 & 12 & 25 \\
16 & 5 & 3 & 8 \\
17 & 2 & 3 & 5 \\
18 & 1 & 0 & 1 \\
& 187 & 185 & 372 \\
\hline
\end{tabular}




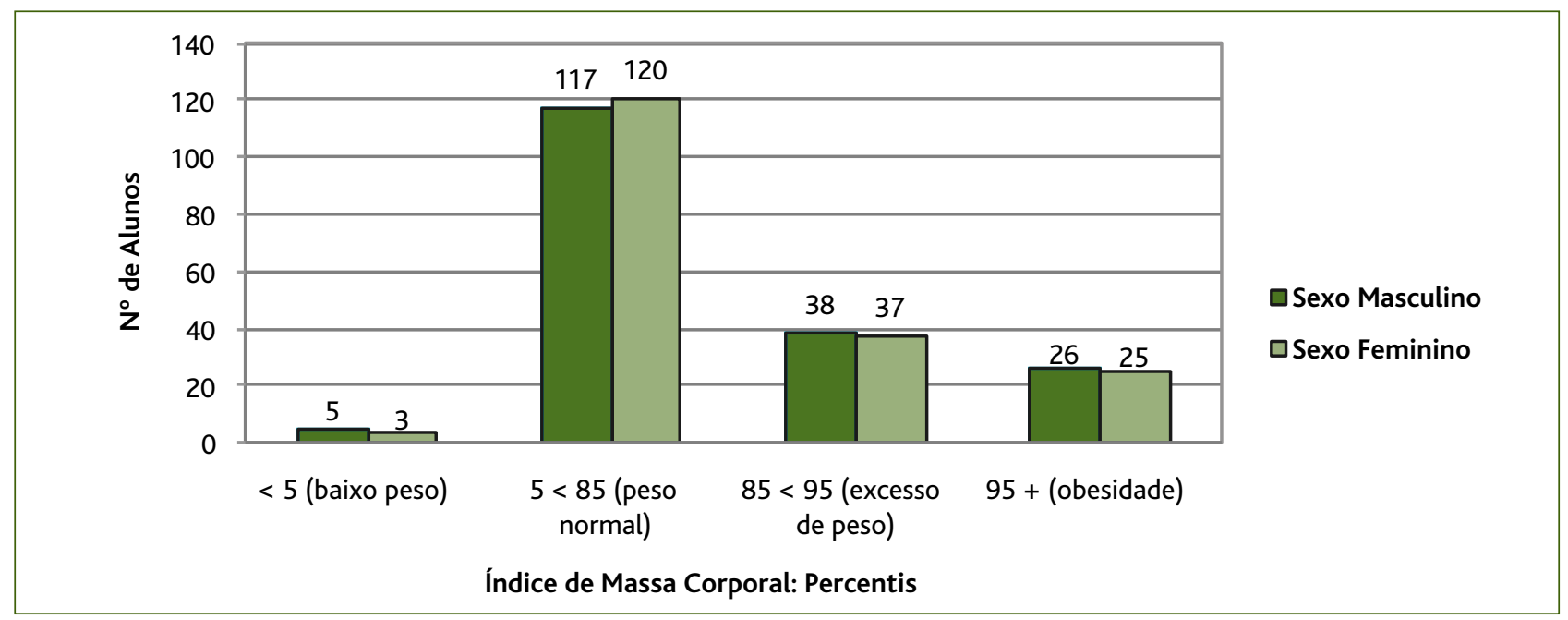

Figura 1. Classes de Índice de massa corporal: classificação por percentis e por sexo.

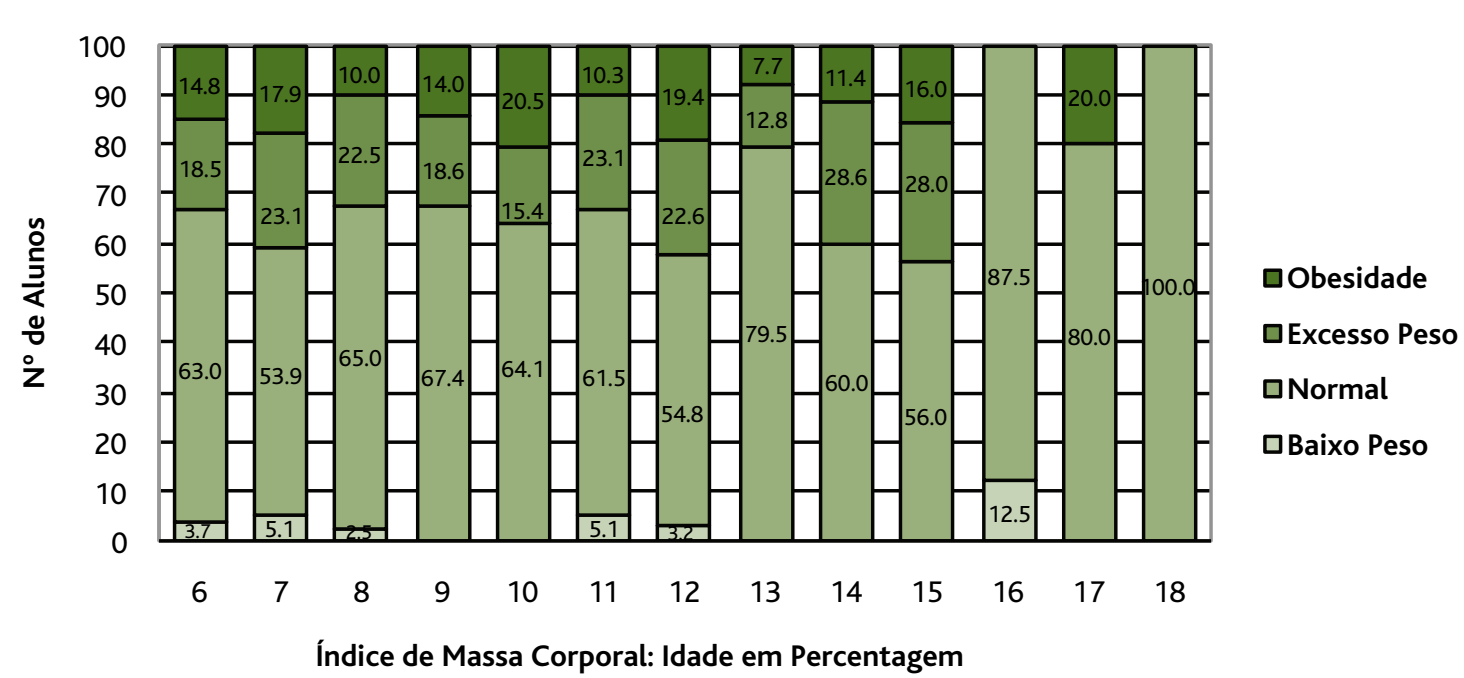

Figura 2. Classes de Índice de massa corporal: classificação por idade, em percentagem.

Verificou-se também que a frequência do peso excessivo variava com a idade (Figura 2), sendo menor na faixa dos 13 anos (20,5\% dos alunos nesse escalão etário) e maior nos alunos de 15 anos $(44,0 \%$ dos alunos desse escalão etário).

Dos 370 alunos com avaliação do perímetro da cintura foram identificados 72 (19,5\%) com obesidade abdominal e 95 (25,7\%) em risco de obesidade abdominal. Estes valores foram mais acentuados no sexo feminino, com metade das alunas apresentando obesidade abdo- minal ou risco de obesidade abdominal (esse número foi de $40,3 \%$ no sexo masculino) e mantiveram-se elevados em todas as idades até aos 15 anos (Figura 3).

\section{DISCUSSÃO}

A prevalência do peso excessivo está de acordo com os resultados da literatura científica existente, ou seja, é uma prevalência muito elevada. Desprezando os dados referentes aos alunos com mais de 15 anos de idade, por serem em número muito reduzido, pode-se con- 


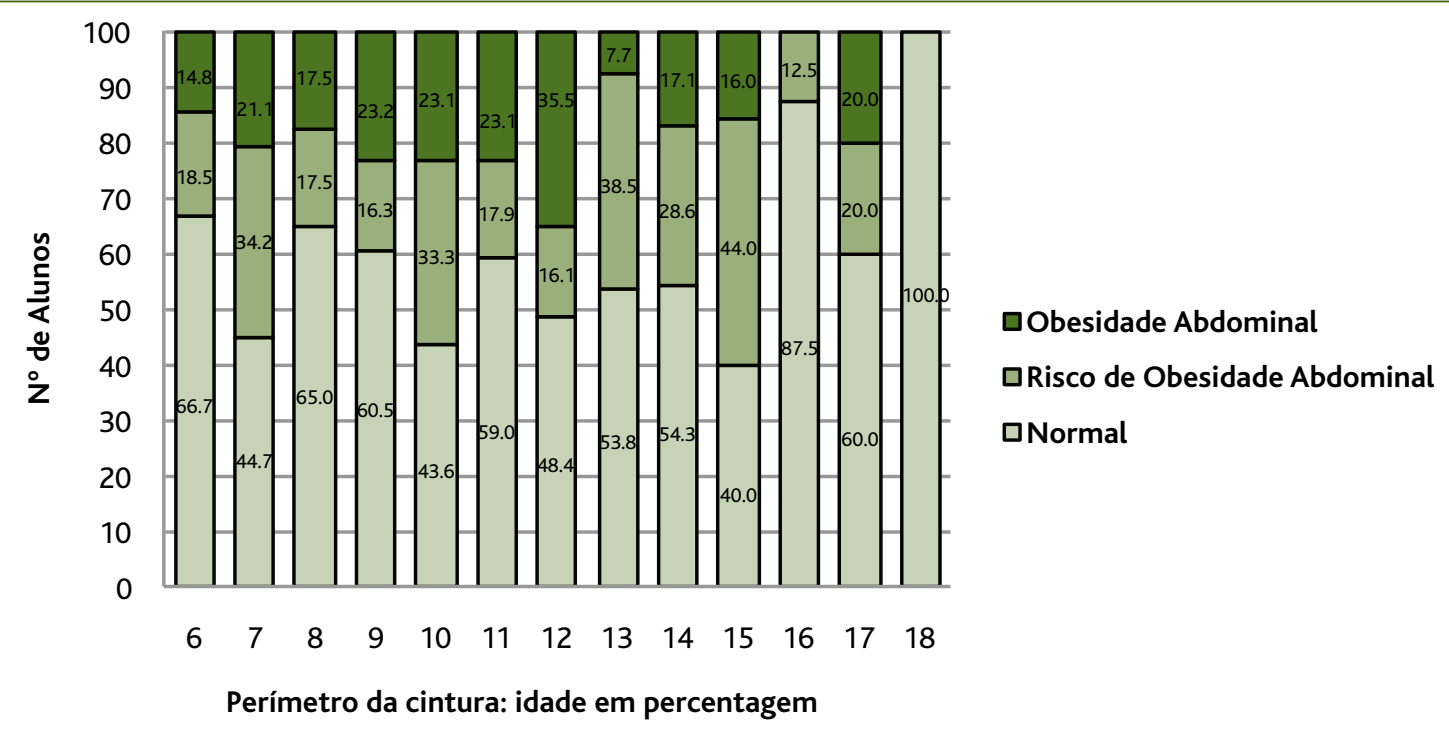

Figura 3. Classes de Índice de massa corporal: classificação por idade, em percentagem.

cluir que é um problema transversal a todas as idades escolares. Já a prevalência da obesidade se revelou bastante superior à encontrada em outros estudos, embora essa comparação seja dificultada por diferenças de objectivos e metodologias.

Padez et al, ${ }^{7}$ observando crianças dos 7 aos 9 anos de idade, encontraram $20,3 \%$ de crianças com excesso de peso e $11,3 \%$ de crianças obesas. Para essa faixa etária, o nosso estudo apresenta, respectivamente, $21,3 \%$ e $13,9 \%$.

Albuquerque, ${ }^{4}$ em crianças com 6 a 12 anos de idade da região Centro, identificou $22,3 \%$ de excesso de peso e $10,7 \%$ de obesidade; Venâncio, ${ }^{6}$ no Lumiar e em crianças do mesmo grupo etário, identificou $15,7 \%$ de excesso de peso e $12,7 \%$ de obesidade. Nas idades dos 6 aos 12 anos encontrámos em Boticas 20,5\% de crianças com excesso de peso e $15,1 \%$ com obesidade.

A OMS, num relatório de $2014,{ }^{9}$ apresenta dados de 2009/2010 referindo que $32,0 \%$ das crianças portuguesas com 11 anos de idade apresentavam excesso de peso ou obesidade, comparável com os $33,4 \%$ no nosso estudo.

A análise para o Global Burden of Disease Study 2013, ${ }^{10}$ refere, para a população portuguesa menor de 20 anos, uma prevalência de $28,7 \%$ de excesso de peso e de $8,9 \%$ de obesidade no sexo masculino $(20,4 \%$ e $14,0 \%$ no nosso estudo) e uma prevalência de $27,1 \%$ e $10,6 \%$ no sexo feminino (20,0\% e $13,5 \%$ no nosso estudo).
Também nos parece preocupante a prevalência de medidas anormais do perímetro da cintura que atingem quase metade da população escolar e são mais evidentes no sexo feminino. Saliente-se que o estudo de Albuquerque identificou obesidade abdominal em $7,8 \%$ de crianças, contra $19,5 \%$ de obesidade abdominal em toda a população escolar de Boticas e $22,6 \%$ entre os 6 e os 12 anos de idade.

O estudo COSI 2010 apresenta dados relativos ao baixo peso em crianças dos 6 aos 8 anos de idade, $0,6 \%$ para a região Norte e $0,7 \%$ para o total nacional, enquanto, para essas idades, a prevalência de baixo peso na nossa população foi de $3,8 \%$.

Os dados deste estudo comprovam que a obesidade infantil representa um problema de saúde importante no concelho de Boticas. O estudo poderá ser o ponto de partida para o desenvolvimento de programas de intervenção na obesidade infantil.

\section{REFERÊNCIAS BIBLIOGRÁFICAS}

1. Direcção-Geral da Saúde. Consultas de vigilância de saúde infantil e juvenil - Actualização das curvas de crescimento: circular normativa ${ }^{\circ}$ 05/DSMIA, de 21/02/2006. Lisboa: DGS; 2006.

2. World Health Organization. Population-based approaches to childhood obesity prevention. Geneva:WHO; 2012. ISBN 9789241504782

3. Carroquino MJ. Prevalence of overweight and obesity in children and adolescents: fact sheet 2.3. Copenhagen:WHO; ENHIS; 2009. Available 
from: http://www.euro.who.int/_data/assets/pdf_file/0005/96980/ 2.3.-Prevalence-of-overweight-and-obesity-EDITED_layouted_V3.pdf

4. Albuquerque D, Nóbrega C, Samouda H, Manco L. Assessment of obesity and abdominal obesity among Portuguese children. Acta Med Port. 2012;25(3):169-73.

5. Rito Al, Paixão E, Carvalho MA, Ramos C. Childhood obesity surveillance initiative: COSI Portugal 2008. Lisboa: INSA; 2010. ISBN 9789728643607

6. Venâncio P, Aguilar S, Pinto G. Obesidade infantil... um problema cada vez mais actual [Obesity in children in Portugal: a cross-sectional study]. Rev Port Med Geral Fam. 2012;28(6): 410-6. Portuguese

7. Padez C, Fernandes T, Mourão I, Moreira P, Rosado V. Prevalence of overweight and obesity in 7-9-year-old Portuguese children: trends in body mass index from 1970-2002. Am J Hum Biol. 2004;16(6):670-8.

8. Graça P. Portugal - Alimentação saudável em números, 2013. Lisboa: Direcção-Geral da Saúde; 2013.

9. World Health Organization, Regional Office for Europe. Country profiles on nutritional, physical activity and obesity in the 53 WHO European Region Member States: methodology and summary. Copenhagen: WHO; 2013. ISBN 9789289050036

10. Ng M, Fleming T, Robinson M, Thomson B, Graetz N, Margono C, et al. Global, regional, and national prevalence of overweight and obesity in children and adults during 1980-2013: a systematic analysis for the Global Burden of Disease Study 2013. Lancet. 2014;384(9945):766-81.

11. Juonala M, Magnussen CG, Berenson GS, Venn A, Burns TL, Sabin MA, et al. Childhood adiposity, adult adiposity, and cardiovascular risk factors. N Engl J Med. 2011;365(20):1876-85.

12. Pereira J, Matos C. Custos indirectos associados à obesidade em Portugal [Productivity costs associated with obesity in Portugal]. Rev Port Saúde Publ. 2003;3(temático):65-80. Portuguese

13. Plourde G. Managing pediatric obesity, barriers and potential solutions. Can Fam Physician. 2012;58(5):503-5.

14. He M, Evans A. Are parents aware that their children are overweight or obese? Do they care? Can Fam Physician. 2007;53(9):1493-9.

15. Lundahl A, Kidwell KM, Nelson TD. Parental underestimates of child weight: a meta-analysis. Pediatrics. 2014;133(3):e689-703.

16. Lopes L, Santos R, Pereira B, Lopes V. Maternal perception of children weight status. Child Care Health Dev. 2013;39(5):728-36.

17. August GP, Caprio S, Fennoy I, Freemark M, Kaufman FR, Lustig RH, et al. Prevention and treatment of pediatric obesity: an endocrine society clinical practice guideline based on expert opinion. J Clin Endocrinol Metab. 2008;93(12):4576-99.

18. Oude Luttikhuis H, Baur L, Jansen H, Shrewsbury VA, O'Malley C, Stolk $\mathrm{RP}$, et al. Interventions for treating obesity in children. Cochrane Database Syst Rev. 2009;(1):CD001872.

19. Barlow SE, Expert Committee. Expert committee recommendations regarding the prevention, assessment and treatment of child and adolescent overweight and obesity: summary report. Pediatrics. 2007;120 Suppl 4;S164-92.

20. Costa CD, Ferreira MG, Amaral R. Obesidade infantil e juvenil [Childhood obesity]. Acta Med Port. 2010;23(3):379-84. Portuguese

21. Waters E, de Silva-Sanigorsky A, Burford BJ, Brown T, Campbell KJ, Gao $Y$, et al. Interventions for preventing obesity in children. Cochrane Database Syst Rev. 2001;(12):CD001871.
22. Rogovik AL, Goldman RD. Pharmacologic treatment of pediatric obesity. Can Fam Physician. 2011;57(2):195-7.

23. Whitlock EP, O'Conner EA, Williams SB, Beil TL, Lutz KW. Effectiveness of primary care interventions for weight management in children and adolescents: an updated, targeted systematic review for the USPSTF. Rockville, MD:Agency for Healthcare Research and Quality; 2010. Available from: http://www.ncbi.nlm.nih.gov/books/NBK36416/

24. Kirk S, Zeller M, Claytor R, Santangelo M, Khoury PR, Daniels SR. The relationship of health outcomes to improvements in BMI in children and adolescents. Obes Res. 2005;13(5):876-82.

25. Kolsgaard ML, Joner G, Brunborg C, Anderssen SA, Tonstad S, Andersen LF. Reduction in BMI z-score and improvement in cardiometabolic risk factors in obese children and adolescents. The Oslo Adiposity Intervention Study: a hospital/public health nurse combined treatment. BMC Pediatrics. 2001;11:47.

26. Cai L, Wu Y, Wilson RF, Seagal JB, Kim MT, Wang Y. Effect of childhood obesity prevention programs on blood pressure: a systematic review and meta-analysis. Circulation. 2014;129(18):1832-9.

27. Plataforma Contra a Obesidade. Direcção Geral da Saúde. Lisboa. Janeiro 2007.

28. Freedman DS, Kahn HS, Mei Z, Grummer-Strawn LM, Dietz WH, Srinivasan SR, et al. Relation of body mass index and waist-to-height ratio to cardiovascular disease risk factors in children and adolescents: the Bogalusa Heart Study. Am J Clin Nutr. 2007;86(1):33-40.

29. Rito A, Breda J, Carmo I. Guia de avaliação do estado nutricional infantil e juvenil. Lisboa: INSA; 2011. ISBN 9789728643614

30. Sardinha LB, Santos R, Vale S, Coelho e Silva MJ, Raimundo AM, Moreira $\mathrm{H}$, et al.Waist circumference percentiles for Portuguese children and adolescents aged 10 to 18 years. Eur J Pediatr. 2012;171(3):499-505.

31. Despacho $n^{\circ} 12.045 / 2006$, de 7 de Junho. Diário da República. $2^{a}$ Série(110).

32. Fernández JR, Redden DT, Pietrobelli A, Allison DB. Waist circumference percentiles in nationally representative samples of African-American, European-American, and Mexican-American children and adolescents. J Pediatr. 2004;145(4):439-44.

\section{CONFLITOS DE INTERESSES}

Os autores declaram não existirem conflitos de interesses na elaboração deste estudo. $\mathrm{O}$ estudo não tem fins lucrativos e não teve fontes de financiamento próprias, contando com o apoio logístico do Centro de Saúde de Boticas.

\section{COMISSÃO DE ÉTICA}

Estudo realizado com parecer favorável da Comissão de Ética para a Saúde da ARS Norte.

\section{ENDEREÇO PARA CORRESPONDÊNCIA}

José Pedro Verdelho Alves

Centro de Saúde de Boticas

Av. Do Eiró - 5460 - 320 Boticas

zepvalves@gmail.com

Recebido em 05-09-2014

Aceite para publicação em 26-01-2015 


\section{ABSTRACT}

\section{ANTHROPOMETRIC EVALUATION OF SCHOOL CHILDREN IN BOTICAS}

Objectives: To determine the prevalence of underweight, overweight, obesity, the risk of abdominal obesity and established abdominal obesity of school children in Boticas.

Type of study: Cross-sectional study.

Setting: Schools in Boticas, Portugal.

Population: All students in Boticas in the first nine years of school in 2012-2013.

Methods: The weight, height, and body mass index of the children were measured and compared with standard growth charts. Waist circumference was measured and compared with the charts of Fernandez et al.

Results: In 2012-2013, 372 students attended the Gomes Monteiro Schools in Boticas. There was a prevalence of $19.7 \%$ of overweight children, with a male predominance. Obesity was found in $12.2 \%$ of students, with a female predominance. There was risk of abdominal obesity and established abdominal obesity in $45.1 \%$ of students, with a female predominance.

Conclusions: Childhood obesity is a major health problem in Boticas, with a prevalence found in this study similar to that reported in the literature. This study could be the starting point for the development of an intervention program for childhood obesity.

Keywords: Overweight; Childhood Obesity; Body Mass Index; Waist Circumference. 